Article

\title{
Synthesis of $\delta$-Oxo-1,1-bis(triflyl)alkanes and Their Acidities
}

Hikaru Yanai ${ }^{1, *}$, Masaya Fujita ${ }^{1}$, Arata Takahashi ${ }^{1}$, Min Zhang ${ }^{2}$, Masaaki Mishima ${ }^{2}$, Akira Kotani ${ }^{1}$, Takashi Matsumoto ${ }^{1}$ and Takeo Taguchi ${ }^{1}$

1 School of Pharmacy, Tokyo University of Pharmacy and Life Sciences, 1432-1 Horinouchi, Hachioji, Tokyo 192-0392, Japan

2 Institute for Materials Chemistry and Engineering, Kyushu University, 6-10-1 Hakozaki, Higashi-ku, Fukuoka 812-8581, Japan

* Author to whom correspondence should be addressed; E-Mail: yanai@toyaku.ac.jp; Tel.: +81-42-676-3265; Fax: +81-42-676-3257.

Received: 4 November 2013; in revised form: 3 December 2013 / Accepted: 11 December 2013 / Published: 13 December 2013

\begin{abstract}
The reaction of 1,1-bis(triflyl)ethylene generated in situ with enolizable carbonyls yielded $\delta$-oxo-1,1-bis(triflyl)alkane derivatives. Their acidities in both the gas and solution phases were determined.
\end{abstract}

Keywords: carbon acid; triflyl group; gas phase acidity; $\mathrm{p} K_{\mathrm{a}}$

\section{Introduction}

The bis(triflyl)methyl $\left(\mathrm{Tf}_{2} \mathrm{CH}\right.$; $\left.\mathrm{Tf}=\mathrm{CF}_{3} \mathrm{SO}_{2}\right)$ group is known to be a strong $\mathrm{C}-\mathrm{H}$ acidic functionality due to the gem-disubstitution of a carbon atom by two triflyl groups. This type of carbon acid $(\mathrm{C}-\mathrm{H}$ acid) shows notably strong acidity not only in the gas-phase [1,2] but also in solution-phase [3]. For example, the gas-phase acidity $\Delta G_{\text {acid }}$ of $\mathrm{Tf}_{2} \mathrm{CH}_{2}(\mathbf{1})$ has been determined to be $300.6 \mathrm{kcal} \mathrm{mol}^{-1}$. Compared to the value of sulfuric acid $\left(302.2 \mathrm{kcal} \mathrm{mol}^{-1}\right)$, this somewhat lower value means that $\mathrm{Tf}_{2} \mathrm{CH}_{2} \mathbf{1}$ performs as a superacid in the gas-phase. The $\mathrm{p} K_{\mathrm{a}}$ of $\mathbf{1}$ in DMSO is also measured as 2.1 and it works as a better proton donor relative to trifluoroacetic acid $\left(\mathrm{p} K_{\mathrm{a}}\right.$ in $\left.\mathrm{DMSO}=3.45\right)$. On the basis of this feature, some powerful Brønsted acid catalysts containing $\mathrm{Tf}_{2} \mathrm{CH}$ functionalities such as $\mathrm{Tf}_{2} \mathrm{CHC}_{6} \mathrm{~F}_{5}$ [4-6], $\mathrm{Tf}_{2} \mathrm{CHCH}_{2} \mathrm{CHTf}_{2}$ [7-10], and multiple carbon acids [11-13] were developed. Compared to the corresponding nitrogen acid $\mathrm{Tf}_{2} \mathrm{NH}$ and oxygen acid $\mathrm{TfOH}$, these carbon acids show excellent catalyst performance in several synthetic reactions, including the Mukaiyama aldol reaction, 
the Friedel-Crafts acylation, and esterification. However, the synthesis and purification of such strongly acidic carbon acids are not so easy [14]. For example, Koshar and co-workers reported that in situ-formation of 1,1-bis(triflyl)ethylene (2) by the reaction of $\mathrm{Tf}_{2} \mathrm{CH}_{2}$ (1) with paraformaldehyde in the presence of $\mathrm{CaSO}_{4}$ and the subsequent one-pot reaction with diethyl malonate (3a) gave the bis(triflyl)ethylated malonate $\mathbf{4 a}$ in poor yield (Scheme 1) [15].

Scheme 1. Koshar's synthesis of bis(triflyl)ethylated malonate 4a.



Since this reaction required harsh conditions for the effective generation of the alkene intermediate $\mathbf{2}$, the yield of $\mathbf{4 a}$ was not very high. To overcome this problem, we reported that 1,1,3,3tetrakis(triflyl)propane (5, Figure 1) [16,17] can be used as not only an acid catalyst, but also a very effective reagent for in situ-generation of $\mathrm{Tf}_{2} \mathrm{C}=\mathrm{CH}_{2}$ (2) via a retro-Michael type reaction. Recently, zwitterion 6 (Figure 1) was also developed for the same use [18]. These reagents can be easily prepared on multi-gram scale from commercially available $\mathrm{Tf}_{2} \mathrm{CH}_{2}(\mathbf{1})$ in one step.

Figure 1. Structures of 1,1-bis(triflyl)ethylating reagents.
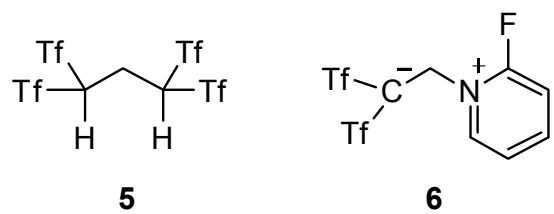

Herein we report an improved synthesis of $\delta$-oxo-1,1-bis(triflyl)alkanes via bis(triflyl)ethylation reaction of enolizable carbonyls with tetrasulfone 5. Furthermore, both gas-phase acidity and $\mathrm{p} K_{\mathrm{a}}$ values in a DMSO solution of some of the prepared carbon acids were determined.

\section{Results and Discussion}

\subsection{Improved Synthesis of $\delta$-Oxo-1,1-bis(triflyl)alkanes}

Keeping Koshar's original work in mind, we first examined the 2,2-bis(triflyl)ethylation reaction of diethyl malonate (3a, Scheme 2). Notably, the reaction of 3a with $\mathrm{Tf}_{2} \mathrm{CHCH}_{2} \mathrm{CHTf}_{2}$ (5) was smoothly completed within $3 \mathrm{~h}$ at $80{ }^{\circ} \mathrm{C}$. In this case, we observed complete consumption of tetrasulfone 5 and quantitative formation of $\mathrm{Tf}_{2} \mathrm{CH}_{2}(\mathbf{1})$ and the desired carbon acid $\mathbf{4 a}$ by ${ }^{19} \mathrm{~F}-\mathrm{NMR}$ analysis of the crude mixture. This mixture was successfully purified by bulb-to-bulb distillation $\left(150{ }^{\circ} \mathrm{C}\right.$ at $5 \mathrm{mmHg}$ ) using a Kugelrohr oven to give acceptably pure carbon acid 4a in $84 \%$ yield. Compared to Koshar's procedure, the use of tetrasulfone 5 instead of $\mathrm{Tf}_{2} \mathrm{CH}_{2} /$ paraformaldehyde resulted in a better yield of $\mathbf{4 a}$ $(50 \%$ vs. $84 \%)$ within a shorter reaction time. 
Scheme 2. Improved synthesis of bis(triflyl)ethylated malonate 4a.

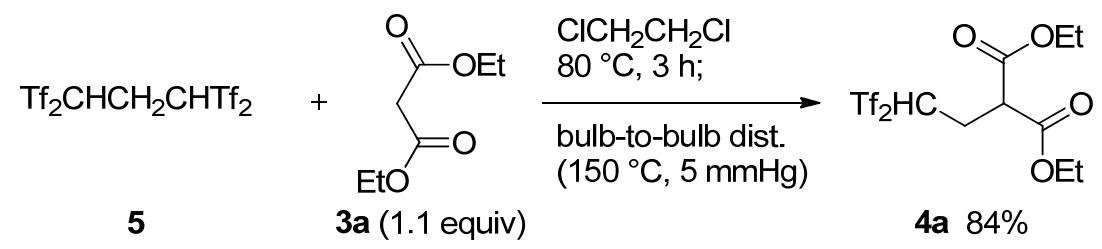

Smooth formation of the carbon acid $\mathbf{4 a}$ in the present case could be attributed to rapid formation of alkene intermediate $\mathbf{2}$ in solution phase from tetrasulfone 5. For instance, ${ }^{1} \mathrm{H}-\mathrm{NMR}$ analysis of a solution of 5 in $\mathrm{CDCl}_{3}$ at $40{ }^{\circ} \mathrm{C}$ revealed very rapid formation of $\mathbf{2}$ in a reversible manner (Figure 2). When this mixture was left for $20 \mathrm{~min}$ at $40{ }^{\circ} \mathrm{C}$, tetrasulfone 5 partly decomposed to $\mathrm{Tf}_{2} \mathrm{CH}_{2}(\mathbf{1})$ and alkene $\mathbf{2}$ to give an equilibrium mixture between $\mathbf{5}$ and $\mathbf{1} / \mathbf{2}$ without formation of any side products and its equilibrium constant $K_{\text {eq }}$ was calculated as $3.21 \times 10^{-3}$.

Figure 2. Reaction profile in a $0.01 \mathrm{M}$ solution of $\mathrm{Tf}_{2} \mathrm{CHCH}_{2} \mathrm{CHTf}_{2}(\mathbf{5})$ in $\mathrm{CDCl}_{3}$ at $40{ }^{\circ} \mathrm{C}$ (squares, $\mathrm{Tf}_{2} \mathrm{CHCH}_{2} \mathrm{CHTf}_{2}$ (5); diamonds, $\mathrm{Tf}_{2} \mathrm{CH}_{2}$ (1); triangles, $\mathrm{Tf}_{2} \mathrm{C}=\mathrm{CH}_{2}$ (2)).

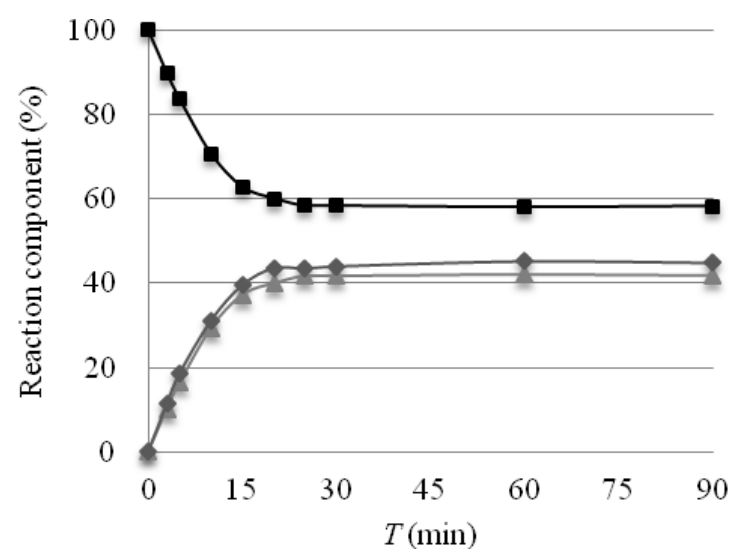

Since the product yield in the bis(triflyl)ethylation with $\mathrm{Tf}_{2} \mathrm{CHCH}_{2} \mathrm{CHTf}_{2}(\mathbf{5})$ was better than that in Koshar's original procedure using $\mathrm{Tf}_{2} \mathrm{CH}_{2}(\mathbf{1})$ and paraformaldehyde, we carried out the reaction of several active methylenes with tetrasulfone 5. Selected results are summarized in Table 1. When some dialkyl malonates such as $\mathbf{3 b}$ and $\mathbf{3 c}$ were treated with tetrasulfone $\mathbf{5}$ at $80{ }^{\circ} \mathrm{C}$ in 1,2-dichloroethane, the desired bis(triflyl)ethylated products $\mathbf{4 b}$ and $\mathbf{4 c}$ were obtained in $84 \%$ and $98 \%$ yield, respectively (entries 1 and 2). In the case of dimethyl malonate (3b), the product $\mathbf{4 b}$ was isolated after standard bulb-to-bulb distillation (method A). Although distillation of dibenzyl derivative $\mathbf{4 c}$ was problematic due to its high boiling point, acceptably pure $4 \mathbf{c}$ was obtained by removal of $\mathrm{Tf}_{2} \mathrm{CH}_{2}(\mathbf{1})$ and remaining 3c by a Kugelrohr oven $\left(120{ }^{\circ} \mathrm{C}\right.$ at $2 \mathrm{mmHg}$; method B). Under similar conditions, carbon acid 4d derived from phosphonyl acetate $\mathbf{3 d}$ was isolated in $57 \%$ yield using method A (entry 3 ). The reactions of $\beta$-ketoesters and of 1,3-diketones proceeded under more mild conditions in $\mathrm{CH}_{2} \mathrm{Cl}_{2}$. For example, the products $4 \mathbf{e}-\mathbf{g}$ derived from $\beta$-ketoesters were obtained in excellent yields by the reaction at $40{ }^{\circ} \mathrm{C}$ (entries 4-6). Likewise, the reactions of 1,3-diketones $\mathbf{3 h}$ and $\mathbf{3 i}$ with tetrasulfone $\mathbf{5}$ completed at room temperature to give the corresponding products $\mathbf{4 h}$ and $\mathbf{4 i}$ in $74 \%$ and $80 \%$ yields, respectively (entries 7 and 8). 
Table 1. Reaction of $\mathrm{Tf}_{2} \mathrm{CHCH}_{2} \mathrm{CHTf}_{2} 5$ with 1,3-dicarbonyl compound 3.

\begin{tabular}{|c|c|c|c|c|c|c|c|}
\hline & & $\begin{array}{c}\mathrm{Tf}_{2} \mathrm{CHCH}_{2} \mathrm{CHTf}_{2}+ \\
\mathbf{5} \\
\end{array}$ & $\begin{array}{l}\text { EWG } \\
\text { EWG } \\
\mathbf{3}\end{array}$ & & $\widehat{L}_{\text {EWG }}^{\text {EWG }}$ & & \\
\hline Entry & & 3 & Temp. $\left({ }^{\circ} \mathrm{C}\right)$ & Time (h) & Method $^{a}$ & 4 & Yield $^{b}(\%)$ \\
\hline $1^{c}$ & $\mathbf{3 b}$ & $\mathrm{CH}_{2}\left(\mathrm{CO}_{2} \mathrm{CH}_{3}\right)_{2}$ & 80 & 8 & $\mathrm{~A}$ & $4 b$ & 84 \\
\hline $2^{c}$ & $3 c$ & $\mathrm{CH}_{2}\left(\mathrm{CO}_{2} \mathrm{Bn}\right)_{2}$ & 80 & 8 & B & $4 c$ & 98 \\
\hline $3^{c}$ & 3d & $\mathrm{CH}_{2}\left(\mathrm{CO}_{2} \mathrm{CH}_{3}\right) \mathrm{P}(\mathrm{O})\left(\mathrm{OCH}_{3}\right)_{2}$ & 80 & 5.5 & $\mathrm{~A}$ & $4 d$ & 57 \\
\hline 4 & $3 e$ & $\mathrm{CH}_{2}(\mathrm{CO} t-\mathrm{Bu}) \mathrm{CO}_{2} \mathrm{CH}_{3}$ & 40 & 2 & A & $4 e$ & 93 \\
\hline 5 & 3f & $\mathrm{CH}_{2}(\mathrm{CO} t-\mathrm{Bu}) \mathrm{CO}_{2} \mathrm{Et}$ & 40 & 5 & $\mathrm{~A}$ & $4 f$ & 82 \\
\hline 6 & $3 g$ & $\mathrm{CH}_{2}(\mathrm{COPh}) \mathrm{CO}_{2} \mathrm{Et}$ & 40 & 4.5 & B & $4 g$ & 86 \\
\hline 7 & $3 h$ & $\mathrm{CH}_{2}(\mathrm{CO} t-\mathrm{Bu})_{2}$ & Rt & 4 & A & $4 h$ & 73 \\
\hline 8 & $3 \mathbf{i}$ & $\mathrm{CH}_{2}(\mathrm{CO} i-\mathrm{Pr})_{2}$ & $\mathrm{Rt}$ & 2.5 & A & $4 i$ & 80 \\
\hline
\end{tabular}

${ }^{a}$ Method A; Product was isolated by bulb-to-bulb distillation. Method B; Product was purified by distillative removal of $\mathrm{Tf}_{2} \mathrm{CH}_{2} \mathbf{1}$ and remaining 3 using a Kugelrohr oven; ${ }^{b}$ Isolated yield; ${ }^{c}$ Reaction was carried out in 1,2-dichloroethane.

As shown in Scheme 3, it should be noted that less reactive triester $\mathbf{3} \mathbf{j}$ smoothly converted to the corresponding bis(triflyl)ethylated product $\mathbf{4} \mathbf{j}$ in $78 \%$ yield under the present conditions. In this case, we found that the use of acetonitrile instead of 1,2-dichloroethane gives a better yield of $\mathbf{4 j}$.

Scheme 3. Bis(triflyl)ethylation of triester $\mathbf{3 j}$.

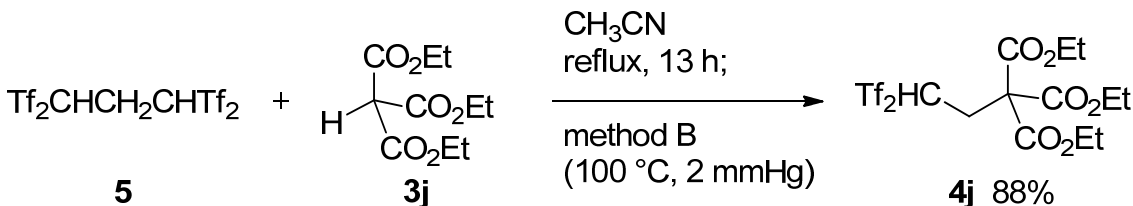

\subsection{Gas-Phase and Solution-Phase Acidities of Carbon Acids}

The gas-phase acidity $\Delta G_{\text {acid }}$ established with the use of the FT-ICR technique [1,2] is known as an extensive scale for strong acids. We measured $\Delta G_{\text {acid }}$ values of some select carbon acids (Table 2). The values of triflylated methanes were reduced by increasing in the number of triflyl group $\left(\mathrm{TfCH}_{3}\right.$, $339.8 \mathrm{kcal} \mathrm{mol}^{-1}$ [19]; $\mathrm{Tf}_{2} \mathrm{CH}_{2}$ (1), $300.6 \mathrm{kcal} \mathrm{mol}^{-1}$ [1]; $\mathrm{Tf}_{3} \mathrm{CH}, 289.0 \mathrm{kcal} \mathrm{mol}^{-1}$ [2]) (entries 1, 2, and 7). The value of $\mathrm{Tf}_{2} \mathrm{CHCH}_{2} \mathrm{CHTf}_{2}$ (5) was recently revised to $290.2 \mathrm{kcal} \mathrm{mol}^{-1}$ and its acidity is notably stronger than that of $\mathrm{Tf}_{2} \mathrm{CH}_{2} \mathbf{1}$ [20]. On the other hand, $\delta$-oxo-1,1-bis(triflyl)alkanes $\mathbf{4 b}, \mathbf{4 e}$, and $\mathbf{4 h}$ showed very similar acidities in gas-phase compared to $\mathrm{Tf}_{2} \mathrm{CH}_{2}(\mathbf{1})$. That is, established $\Delta G_{\text {acid }}$ values of these compounds were $299.6 \mathrm{kcal} \mathrm{mol}^{-1}, 300.3 \mathrm{kcal} \mathrm{mol}^{-1}$, and $300.4 \mathrm{kcal} \mathrm{mol}^{-1}$, respectively (entries 3-5). This finding suggests that the difference of carbonyl functionalities in the structures of $\delta$-oxo-1,1-bis(triflyl)alkanes is not critical factor for their gas-phase acidities. Therefore, the symmetrical structure of $\mathbf{5}$ plays an important role for its significantly enhanced acidity (the statistical effect). In addition, the $\mathrm{p} K_{\mathrm{a}}$ value of carbon acid $\mathbf{4 b}$ in DMSO solution was determined as 2.16 by the voltammetric method [21,22]. This also means that the $\mathbf{4}$ is comparable in the acidity to $\mathbf{1}\left(\mathrm{p} K_{\mathrm{a}}=2.1\right)$ in DMSO solution. 
Table 2. The gas-phase acidities of carbon acids.

\begin{tabular}{ccc}
\hline Entry & Carbon Acid & $\Delta \boldsymbol{G}_{\text {acid }}\left(\mathbf{k c a l ~ m o l} \mathbf{~ m}^{\mathbf{- 1}}\right)$ \\
\hline $1^{a}$ & $\mathrm{TfCH}_{3}$ & 339.8 \\
$2^{b}$ & $\mathrm{Tf}_{2} \mathrm{CH}_{2}(\mathbf{1})$ & 300.6 \\
3 & $\mathrm{Tf}_{2} \mathrm{CHCH}_{2} \mathrm{CH}(\mathrm{CO} t-\mathrm{Bu})_{2}(\mathbf{4 h})$ & 300.4 \\
4 & $\mathrm{Tf}_{2} \mathrm{CHCH}_{2} \mathrm{CH}(\mathrm{CO} t-\mathrm{Bu}) \mathrm{CO}_{2} \mathrm{CH}_{3}(\mathbf{4 e})$ & 300.3 \\
5 & $\mathrm{Tf}_{2} \mathrm{CHCH}_{2} \mathrm{CH}\left(\mathrm{CO}_{2} \mathrm{CH}_{3}\right)_{2}(\mathbf{4 b})$ & 299.6 \\
$6^{c}$ & $\mathrm{Tf}_{2} \mathrm{CHCH}_{2} \mathrm{CHTf}$ & $(\mathbf{5})$ \\
$7^{d}$ & $\mathrm{Tf}_{3} \mathrm{CH}$ & 290.2 \\
\hline
\end{tabular}

${ }^{a}$ Ref. [19]. ${ }^{b}$ Ref. [1]. ${ }^{c}$ Ref. [20]. ${ }^{d}$ Ref. [2].

\section{Experimental}

\subsection{General}

All reactions were carried out under Ar atmosphere. Melting points were uncorrected. ${ }^{1} \mathrm{H}-(400 \mathrm{MHz})$ and ${ }^{13} \mathrm{C}-\mathrm{NMR}(100 \mathrm{MHz})$ spectra were taken on a Bruker DPX 400 spectrometer, and chemical shifts were reported in parts per million (ppm) using $\mathrm{CHCl}_{3}\left(7.26 \mathrm{ppm}\right.$ ) in $\mathrm{CDCl}_{3}$ for ${ }^{1} \mathrm{H}-\mathrm{NMR}$, and $\mathrm{CDCl}_{3}$ (77.01 ppm) for ${ }^{13} \mathrm{C}$-NMR as an internal standard, respectively. ${ }^{19} \mathrm{~F}-\mathrm{NMR}$ spectra were taken on a Varian Mercury 300 spectrometer $\left(282 \mathrm{MHz}\right.$ for $\left.{ }^{19} \mathrm{~F}\right)$, and chemical shifts were reported in parts per million using trifluoromethylbenzene $(0 \mathrm{ppm})$ as a standard. Mass spectra were recorded by an electrospray ionization-time of flight (ESI-TOF) mass spectrometer (Micromass LCT). IR spectra were recorded by a JASCO FT/IR 4100 spectrometer. Column chromatography was performed on neutral silica gel (75-150 $\mu \mathrm{m})$. $\mathrm{Tf}_{2} \mathrm{CHCH}_{2} \mathrm{CHTf}_{2}(\mathbf{5})$ was prepared from $\mathrm{Tf}_{2} \mathrm{CH}_{2}(\mathbf{1})$ by the reported procedure [15].

\subsection{General Procedure for Bis(triflyl)ethylation Reaction of Enolizable Carbonyls}

To a solution of carbonyl compound (1.0-2.0 equiv) in $\mathrm{CH}_{2} \mathrm{Cl}_{2}$ or 1,2-dichloroethane, $\mathrm{Tf}_{2} \mathrm{CHCH}_{2} \mathrm{CHTf}_{2}$ $(5,0.50 \mathrm{mmol})$ was added at room temperature. After stirring at room temperature to $80{ }^{\circ} \mathrm{C}$, the reaction mixture was concentrated under reduced pressure. The resultant residue was purified by bulb-to-bulb distillation using a Kugelrohr oven to give bis(triflyl)ethylated product 4.

Diethyl 2-(2,2-bis(trifluoromethylsulfonyl)ethyl)malonate (4a). According to the general procedure, this compound was obtained in $84 \%$ yield $(190 \mathrm{mg}, 0.420 \mathrm{mmol})$ by the reaction of $\mathrm{Tf}_{2} \mathrm{CHCH}_{2} \mathrm{CHTf}_{2}$ (5, $286 \mathrm{mg}, 0.500 \mathrm{mmol})$ with diethyl malonate $(83.5 \mu \mathrm{L}, 0.55 \mathrm{mmol})$ in $\mathrm{CH}_{2} \mathrm{Cl}_{2}(0.10 \mathrm{~mL})$ at $80{ }^{\circ} \mathrm{C}$ for $3 \mathrm{~h}$ and the following bulb-to-bulb distillation $\left(140-160{ }^{\circ} \mathrm{C}\right.$ at $5 \mathrm{mmHg}$ ). Colorless oil; IR (neat) $v$ 2989, 2944, 1746, 1397, 1214, 1115, $1024 \mathrm{~cm}^{-1}$; ${ }^{1} \mathrm{H}-\mathrm{NMR}\left(\mathrm{CDCl}_{3}\right) \delta 1.29(6 \mathrm{H}, \mathrm{t}, J=7.1 \mathrm{~Hz}), 2.91-2.98$ $(2 \mathrm{H}, \mathrm{m}), 3.93(1 \mathrm{H}, \mathrm{t}, J=7.8 \mathrm{~Hz}), 4.19-4.33(4 \mathrm{H}, \mathrm{m}), 5.73(1 \mathrm{H}, \mathrm{t}, J=6.6 \mathrm{~Hz}) ;{ }^{13} \mathrm{C}-\mathrm{NMR}\left(\mathrm{CDCl}_{3}\right) \delta$ 13.9, 24.6, 47.5, 62.7, 74.4, 119.2 (q, $\left.J_{\mathrm{C}-\mathrm{F}}=329.6 \mathrm{~Hz}\right), 167.5 ;{ }^{19} \mathrm{~F}-\mathrm{NMR}\left(\mathrm{CDCl}_{3}\right) \delta-10.1(6 \mathrm{~F}, \mathrm{~s})$; MS (ESI-TOF) $m / z 453[\mathrm{M}+\mathrm{H}]^{+}$; HRMS calcd. for $\mathrm{C}_{11} \mathrm{H}_{15} \mathrm{~F}_{6} \mathrm{O}_{8} \mathrm{~S}_{2}[\mathrm{M}+\mathrm{H}]^{+}, 453.0113$; found, 453.0054 . Anal. calcd. for $\mathrm{C}_{11} \mathrm{H}_{14} \mathrm{~F}_{6} \mathrm{O}_{8} \mathrm{~S}_{2}$ : C, 29.21; H, 3.12. Found: C, 29.24; H, 3.19.

Dimethyl 2-(2,2-bis(trifluoromethylsulfonyl)ethyl)malonate (4b). According to the general procedure, this compound was obtained in $84 \%$ yield $(178 \mathrm{mg}, 0.420 \mathrm{mmol})$ by the reaction of $\mathrm{Tf}_{2} \mathrm{CHCH}_{2} \mathrm{CHTf}_{2}$ 
(5, $286 \mathrm{mg}, 0.500 \mathrm{mmol})$ with dimethyl malonate $(62.9 \mu \mathrm{L}, 0.55 \mathrm{mmol})$ in $\mathrm{CH}_{2} \mathrm{Cl}_{2}(0.20 \mathrm{~mL})$ at $80{ }^{\circ} \mathrm{C}$ for $8 \mathrm{~h}$ and the following bulb-to-bulb distillation $\left(160-170{ }^{\circ} \mathrm{C}\right.$ at $\left.5 \mathrm{mmHg}\right)$. Colorless crystals (Et ${ }_{2} \mathrm{O}$-hexane); Mp. $44.2-46.8^{\circ} \mathrm{C}$; IR (neat) v 3007, 2962, 2854, 1747, 1440, 1395, 1215, 1114, 1036, 1004, $705 \mathrm{~cm}^{-1} ;{ }^{1} \mathrm{H}-\mathrm{NMR}\left(\mathrm{CDCl}_{3}\right) \delta 2.92-2.98(2 \mathrm{H}, \mathrm{m}), 3.81(6 \mathrm{H}, \mathrm{s}), 3.99(1 \mathrm{H}, \mathrm{t}, J=7.8 \mathrm{~Hz}), 5.69(1 \mathrm{H}$, $\mathrm{t}, J=6.6 \mathrm{~Hz}) ;{ }^{13} \mathrm{C}-\mathrm{NMR}\left(\mathrm{CDCl}_{3}\right) \delta 24.7,47.3,53.5,74.3,119.2\left(\mathrm{q}, J_{\mathrm{C}-\mathrm{F}}=329.8 \mathrm{~Hz}\right), 167.8 ;{ }^{19} \mathrm{~F}-\mathrm{NMR}$ $\left(\mathrm{CDCl}_{3}\right) \delta-10.0(6 \mathrm{~F}, \mathrm{~s})$; MS (ESI-TOF) $\mathrm{m} / z 425[\mathrm{M}+\mathrm{H}]^{+}$; HRMS calcd for $\mathrm{C}_{9} \mathrm{H}_{11} \mathrm{~F}_{6} \mathrm{O}_{8} \mathrm{~S}_{2}[\mathrm{M}+\mathrm{H}]^{+}$, 424.9800; found, 424.9825. Anal. calcd. for $\mathrm{C}_{9} \mathrm{H}_{10} \mathrm{~F}_{6} \mathrm{O}_{8} \mathrm{~S}_{2}$ : C, 25.48; H, 2.38. Found: C, 25.82; H, 2.57.

Dibenzyl 2-(2,2-bis(trifluoromethylsulfonyl)ethyl)malonate (4c). According to the general procedure, this compound was obtained in $98 \%$ yield $(56.7 \mathrm{mg}, 984 \mu \mathrm{mol})$ by the reaction of dibenzyl malonate (28.4 mg, $0.10 \mathrm{mmol})$ with $\mathrm{Tf}_{2} \mathrm{CHCH}_{2} \mathrm{CHTf}_{2}(\mathbf{5}, 64.0 \mathrm{mg}, 0.112 \mathrm{mmol})$ in $\mathrm{CH}_{2} \mathrm{Cl}_{2}(0.15 \mathrm{~mL})$ at $80{ }^{\circ} \mathrm{C}$ for $8 \mathrm{~h}$ and the following removal of $\mathrm{Tf}_{2} \mathrm{CH}_{2}$ using a Kugelrohr oven $\left(140-150{ }^{\circ} \mathrm{C}\right.$ at $5 \mathrm{mmHg}$ ). Colorless oil; IR (neat) $v$ 3068, 3036, 2954, 1747, 1498, 1456, 1396, 1302, 1215, 1114, 750, $697 \mathrm{~cm}^{-1}$; ${ }^{1} \mathrm{H}-\mathrm{NMR}\left(\mathrm{CDCl}_{3}\right) \delta 2.98(2 \mathrm{H}, \mathrm{t}, J=7.2 \mathrm{~Hz}), 4.05(1 \mathrm{H}, \mathrm{t}, J=7.2 \mathrm{~Hz}), 5.15(2 \mathrm{H}, \mathrm{d}, J=12.3 \mathrm{~Hz}), 5.18(2 \mathrm{H}$, $\mathrm{d}, J=12.3 \mathrm{~Hz}), 5.66(1 \mathrm{H}, \mathrm{t}, J=7.2 \mathrm{~Hz}), 7.24-7.27(4 \mathrm{H}, \mathrm{m}), 7.30-7.34(6 \mathrm{H}, \mathrm{m}) ;{ }^{13} \mathrm{C}-\mathrm{NMR}\left(\mathrm{CDCl}_{3}\right) \delta$ $24.5,47.6,68.3,74.3,119.2\left(\mathrm{q}, J_{\mathrm{C}-\mathrm{F}}=329.7 \mathrm{~Hz}\right), 128.4,128.7,128.8,134.3,167.2 ;{ }^{19} \mathrm{~F}-\mathrm{NMR}\left(\mathrm{CDCl}_{3}\right)$ $\delta-10.0(6 \mathrm{~F}, \mathrm{~s})$; MS (ESI-TOF) $m / z 599[\mathrm{M}+\mathrm{Na}]^{+}$; HRMS calcd. for $\mathrm{C}_{21} \mathrm{H}_{18} \mathrm{~F}_{6} \mathrm{NaO}_{8} \mathrm{~S}_{2}[\mathrm{M}+\mathrm{Na}]^{+}$, 599.0245; found, 599.0247. Anal. calcd. for $\mathrm{C}_{21} \mathrm{H}_{18} \mathrm{~F}_{6} \mathrm{O}_{8} \mathrm{~S}_{2}$ : C, 43.75; H, 3.15. Found: C, 44.09; H, 3.35.

Methyl 2-(dimethoxyphosphoryl)-4,4-bis(trifluoromethylsulfonyl)butanoate (4d). According to the general procedure, this compound was obtained in $57 \%$ yield $(68.2 \mathrm{mg}, 0.144 \mathrm{mmol})$ by the reaction of $\mathrm{Tf}_{2} \mathrm{CHCH}_{2} \mathrm{CHTf}_{2}(\mathbf{5}, 145 \mathrm{mg}, 0.253 \mathrm{mmol})$ with methyl 2-(dimethoxyphosphoryl)acetate (47.2 $\mathrm{mg}$, $0.259 \mathrm{mmol})$ in 1,2-dichloroethane $(0.40 \mathrm{~mL})$ at $80{ }^{\circ} \mathrm{C}$ for $5.5 \mathrm{~h}$ and the following bulb-to-bulb distillation $\left(190-210{ }^{\circ} \mathrm{C}\right.$ at $\left.5 \mathrm{mmHg}\right)$. Colorless crystal $\left(\mathrm{Et}_{2} \mathrm{O}\right)$; Mp. 80.5-83.2 ${ }^{\circ} \mathrm{C}$; IR (neat) $v$ 2964, 1741, 1394, 1342, 1216, 1115, $1053 \mathrm{~cm}^{-1} ;{ }^{1} \mathrm{H}-\mathrm{NMR}\left(\mathrm{CDCl}_{3}\right) \delta 2.86-3.04(2 \mathrm{H}, \mathrm{m}), 3.63(1 \mathrm{H}, \mathrm{dt}$, $\left.J_{\mathrm{H}-\mathrm{P}}=23.8 \mathrm{~Hz}, J_{\mathrm{H}-\mathrm{H}}=7.8 \mathrm{~Hz}\right), 3.818(3 \mathrm{H}, \mathrm{s}), 3.821\left(3 \mathrm{H}, \mathrm{d}, J_{\mathrm{H}-\mathrm{P}}=10.9 \mathrm{~Hz}\right), 3.85\left(3 \mathrm{H}, \mathrm{d}, J_{\mathrm{H}-\mathrm{P}}=10.5\right.$ $\mathrm{Hz}), 6.21(1 \mathrm{H}, \mathrm{t}, J=6.4 \mathrm{~Hz}) ;{ }^{13} \mathrm{C}-\mathrm{NMR}\left(\mathrm{CDCl}_{3}\right) \delta 23.2,40.1\left(\mathrm{~d}, J_{\mathrm{C}-\mathrm{P}}=130.5 \mathrm{~Hz}\right), 53.5,53.9(\mathrm{~d}$, $\left.J_{\mathrm{C}-\mathrm{P}}=7.1 \mathrm{~Hz}\right), 54.0\left(\mathrm{~d}, J_{\mathrm{C}-\mathrm{P}}=6.4 \mathrm{~Hz}\right), 74.5,119.2\left(\mathrm{~d}, J_{\mathrm{C}-\mathrm{F}}=329.8 \mathrm{~Hz}\right), 167.3\left(\mathrm{~d}, J_{\mathrm{C}-\mathrm{P}}=6.9 \mathrm{~Hz}\right)$; ${ }^{19} \mathrm{~F}-\mathrm{NMR}\left(\mathrm{CDCl}_{3}\right) \delta-10.3(3 \mathrm{~F}, \mathrm{~s}),-10.0(3 \mathrm{~F}, \mathrm{~s})$; MS (ESI-TOF) $\mathrm{m} / z 475[\mathrm{M}+\mathrm{H}]^{+}$; HRMS calcd. for $\mathrm{C}_{9} \mathrm{H}_{14} \mathrm{~F}_{6} \mathrm{O}_{9} \mathrm{PS}_{2}[\mathrm{M}+\mathrm{H}]^{+}$, 474.9721; found, 474.9714. Anal. calcd. for $\mathrm{C}_{9} \mathrm{H}_{13} \mathrm{~F}_{6} \mathrm{O}_{9} \mathrm{PS}_{2}: \mathrm{C}, 22.79 ; \mathrm{H}, 2.76$. Found: C, 22.63; H, 3.04.

Methyl 2-(2,2-bis(trifluoromethylsulfonyl)ethyl)-4,4-dimethyl-3-oxopentanoate (4e). According to the general procedure, this compound was obtained in $93 \%$ yield $(73.4 \mathrm{mg}, 0.163 \mathrm{mmol})$ by the reaction of $\mathrm{Tf}_{2} \mathrm{CHCH}_{2} \mathrm{CHTf}_{2}(5,100 \mathrm{mg}, 0.175 \mathrm{mmol})$ with methyl 4,4-dimethyl-3-oxopentanoate $(31 \mu \mathrm{L}$, $0.19 \mathrm{mmol})$ in $\mathrm{CH}_{2} \mathrm{Cl}_{2}(0.15 \mathrm{~mL})$ at $40{ }^{\circ} \mathrm{C}$ for $2 \mathrm{~h}$ and the following bulb-to-bulb distillation $\left(150-160{ }^{\circ} \mathrm{C}\right.$ at $5 \mathrm{mmHg}$ ). Colorless crystals $\left(\mathrm{Et}_{2} \mathrm{O}-\right.$ hexane); Mp. 55.1-55.9 ${ }^{\circ} \mathrm{C}$; IR $(\mathrm{KBr}) v 2975,2911,2880,1742$, 1712, 1481, 1439, 1396, 1350, 1214, 1115, 970, 699, $676 \mathrm{~cm}^{-1} ;{ }^{1} \mathrm{H}-\mathrm{NMR}\left(\mathrm{CDCl}_{3}\right) \delta 1.21(9 \mathrm{H}, \mathrm{s}), 2.60$ $(1 \mathrm{H}, \mathrm{ddd}, J=15.0,9.6,4.5 \mathrm{~Hz}), 2.95(1 \mathrm{H}, \mathrm{ddd}, J=15.0,10.5,3.6 \mathrm{~Hz}), 3.74(3 \mathrm{H}, \mathrm{s}), 4.53(1 \mathrm{H}, \mathrm{dd}$, $J=10.5,4.5 \mathrm{~Hz}), 5.68(1 \mathrm{H}, \mathrm{dd}, J=9.6,3.6 \mathrm{~Hz}) ;{ }^{13} \mathrm{C}-\mathrm{NMR}\left(\mathrm{CDCl}_{3}\right) \delta 25.8,25.9,45.8,47.3,53.2,74.6$, $119.20\left(\mathrm{q}, J_{\mathrm{C}-\mathrm{F}}=329.8 \mathrm{~Hz}\right), 119.23\left(\mathrm{q}, J_{\mathrm{C}-\mathrm{F}}=329.7 \mathrm{~Hz}\right), 169.2,208.6 ;{ }^{19} \mathrm{~F}-\mathrm{NMR}\left(\mathrm{CDCl}_{3}\right) \delta-10.2(3 \mathrm{~F}$, 
s), -10.1 (3F, s); MS (ESI-TOF) $m / z 451[\mathrm{M}+\mathrm{H}]^{+}$; HRMS calcd. for $\mathrm{C}_{12} \mathrm{H}_{17} \mathrm{~F}_{6} \mathrm{O}_{7} \mathrm{~S}_{2}[\mathrm{M}+\mathrm{H}]^{+}, 451.0320$; found, 451.0320. Anal. calcd. for $\mathrm{C}_{12} \mathrm{H}_{16} \mathrm{~F}_{6} \mathrm{O}_{7} \mathrm{~S}_{2}$ : C, 32.00; H, 3.58. Found: C, 31.63; H, 3.63.

Ethyl 2-(2,2-bis(trifluoromethylsulfonyl)ethyl)-4,4-dimethyl-3-oxopentanoate (4f). According to the general procedure, this compound was obtained in $82 \%$ yield $(223 \mathrm{mg}, 0.480 \mathrm{mmol})$ by the reaction of $\mathrm{Tf}_{2} \mathrm{CHCH}_{2} \mathrm{CHTf}_{2}(\mathbf{5}, 336 \mathrm{mg}, 0.587 \mathrm{mmol})$ with ethyl 4,4-dimethyl-3-oxopentanoate (104 $\left.\mu \mathrm{L}, 0.59 \mathrm{mmol}\right)$ in 1,2-dichloroethane $(0.50 \mathrm{~mL})$ at $40{ }^{\circ} \mathrm{C}$ for $5 \mathrm{~h}$ and the following bulb-to-bulb distillation $\left(160-170{ }^{\circ} \mathrm{C}\right.$ at $5 \mathrm{mmHg})$. Colorless crystals $\left(\mathrm{Et}_{2} \mathrm{O}-\right.$ hexane); Mp. 34.7-36.5 ${ }^{\circ} \mathrm{C}$; IR (KBr) v 2979, 2942, 2911, 2876, 1738, 1712, 1480, 1397, 1350, 1213, 1114, 847, 781, $678 \mathrm{~cm}^{-1} ;{ }^{1} \mathrm{H}-\mathrm{NMR}\left(\mathrm{CDCl}_{3}\right) \delta 1.20(9 \mathrm{H}, \mathrm{s}), 1.24$ $(3 \mathrm{H}, \mathrm{t}, J=7.1 \mathrm{~Hz}), 2.58(1 \mathrm{H}, \mathrm{ddd}, J=15.1,10.3,4.4 \mathrm{~Hz}), 2.95(1 \mathrm{H}, \mathrm{ddd}, J=15.1,11.2,3.5 \mathrm{~Hz}), 4.18$ $(2 \mathrm{H}, \mathrm{q}, J=7.1 \mathrm{~Hz}), 4.50(1 \mathrm{H}, \mathrm{dd}, J=11.2,4.4 \mathrm{~Hz}), 5.71(1 \mathrm{H}, \mathrm{dd}, J=10.3,3.5 \mathrm{~Hz}) ;{ }^{13} \mathrm{C}-\mathrm{NMR}\left(\mathrm{CDCl}_{3}\right)$ $\delta 13.7,25.7,25.9,45.7,47.4,62.5,74.6,119.20$ (q, $\left.J_{\mathrm{C}-\mathrm{F}}=329.6 \mathrm{~Hz}\right), 119.24\left(\mathrm{q}, J_{\mathrm{C}-\mathrm{F}}=329.8 \mathrm{~Hz}\right)$, 168.7, 208.7; ${ }^{19} \mathrm{~F}-\mathrm{NMR}\left(\mathrm{CDCl}_{3}\right) \delta-10.3(3 \mathrm{~F}, \mathrm{~s}),-10.0(3 \mathrm{~F}, \mathrm{~s})$; MS (ESI-TOF) $\mathrm{m} / z \quad 465[\mathrm{M}+\mathrm{H}]^{+}$; HRMS calcd. for $\mathrm{C}_{13} \mathrm{H}_{19} \mathrm{~F}_{6} \mathrm{O}_{7} \mathrm{~S}_{2}[\mathrm{M}+\mathrm{H}]^{+}$, 465.0476; found, 465.0496. Anal. calcd. for $\mathrm{C}_{13} \mathrm{H}_{18} \mathrm{~F}_{6} \mathrm{O}_{7} \mathrm{~S}_{2}$ : C, 33.63; H, 3.91. Found: C, 33.47; H, 4.17.

Ethyl 2-benzoyl-4,4-bis(trifluoromethylsulfonyl)butanoate (4g). According to the general procedure, this compound was obtained in $86 \%$ yield $(86.1 \mathrm{mg}, 0.178 \mathrm{mmol})$ by the reaction of $\mathrm{Tf}_{2} \mathrm{CHCH}_{2} \mathrm{CHTf}_{2}$ (5, $119 \mathrm{mg}, 0.208 \mathrm{mmol}$ ) with ethyl 3-oxo-3-phenylpropanoate $(44.0 \mathrm{mg}, 0.229 \mathrm{mmol})$ in $\mathrm{CH}_{2} \mathrm{Cl}_{2}$ $(0.15 \mathrm{~mL})$ at $40{ }^{\circ} \mathrm{C}$ for $4.5 \mathrm{~h}$ and the following removal of $\mathrm{Tf}_{2} \mathrm{CH}_{2}(\mathbf{1})$ using a Kugelrohr oven $\left(120{ }^{\circ} \mathrm{C}\right.$ at $5 \mathrm{mmHg}$ ). Colorless oil; IR (neat) $v$ 3068, 2990, 2932, 1738, 1688, 1598, 1449, 1396, 1293, 1215, 1114, 1028, 774, $689 \mathrm{~cm}^{-1} ;{ }^{1} \mathrm{H}-\mathrm{NMR}\left(\mathrm{CDCl}_{3}\right) \delta 1.14(3 \mathrm{H}, \mathrm{t}, J=7.1 \mathrm{~Hz}), 2.95-3.08(1 \mathrm{H}, \mathrm{m}), 3.08-3.16$ $(1 \mathrm{H}, \mathrm{ddd}, J=15.8,8.8,5.7 \mathrm{~Hz}), 4.16(2 \mathrm{H}, \mathrm{q}, J=7.1 \mathrm{~Hz}), 5.02(1 \mathrm{H}, \mathrm{dd}, J=8.8,6.4 \mathrm{~Hz}), 5.62(1 \mathrm{H}, \mathrm{dd}$, $J=8.0,5.7 \mathrm{~Hz}), 7.53(2 \mathrm{H}, \mathrm{t}, J=7.0 \mathrm{~Hz}), 7.64-7.68(1 \mathrm{H}, \mathrm{m}), 8.00(2 \mathrm{H}, \mathrm{d}, J=7.0 \mathrm{~Hz}) ;{ }^{13} \mathrm{C}-\mathrm{NMR}$ $\left(\mathrm{CDCl}_{3}\right) \delta 13.7,24.7,49.3,62.7,74.8,119.22\left(\mathrm{q}, J_{\mathrm{C}-\mathrm{F}}=329.8 \mathrm{~Hz}\right), 119.25\left(\mathrm{q}, J_{\mathrm{C}-\mathrm{F}}=329.8 \mathrm{~Hz}\right), 128.9$, 129.0, 134.5, 135.1, 168.1, 193.0; ${ }^{19} \mathrm{~F}-\mathrm{NMR}\left(\mathrm{CDCl}_{3}\right) \delta-10.1(3 \mathrm{~F}, \mathrm{~s}),-9.9(3 \mathrm{~F}, \mathrm{~s})$; MS (ESI-TOF) $m / z$ $485[\mathrm{M}+\mathrm{H}]^{+}$; HRMS calcd. for $\mathrm{C}_{15} \mathrm{H}_{15} \mathrm{~F}_{6} \mathrm{O}_{7} \mathrm{~S}_{2}[\mathrm{M}+\mathrm{H}]^{+}$, 485.0163; found, 485.0156.

4-(2,2-Bis(trifluoromethylsulfonyl)ethyl)-2,2,6,6-tetramethylheptane-3,5-dione (4h). According to the general procedure, this compound was obtained in $73 \%$ yield $(72.8 \mathrm{mg}, 0.153 \mathrm{mmol})$ by the reaction of $\mathrm{Tf}_{2} \mathrm{CHCH}_{2} \mathrm{CHTf}_{2}(\mathbf{5}, 119 \mathrm{mg}, 0.208 \mathrm{mmol})$ with 2,2,6,6-tetramethylheptane-3,5-dione (48 $\left.\mu \mathrm{L}, 0.23 \mathrm{mmol}\right)$ in $\mathrm{CH}_{2} \mathrm{Cl}_{2}(0.30 \mathrm{~mL})$ for $4 \mathrm{~h}$ at room temperature and the following bulb-to-bulb distillation $\left(150-170{ }^{\circ} \mathrm{C}\right.$ at $5 \mathrm{mmHg})$. Colorless crystals $\left(\mathrm{CHCl}_{3}\right)$; Mp. 51.0-52.2 ${ }^{\circ} \mathrm{C}$; IR $(\mathrm{KBr})$ v 2973, 2911, 2877, 1713, 1481, 1398, 1213, 1114, 1152, $677 \mathrm{~cm}^{-1} ;{ }^{1} \mathrm{H}-\mathrm{NMR}\left(\mathrm{CDCl}_{3}\right) \delta 1.22(18 \mathrm{H}, \mathrm{s}), 2.76(2 \mathrm{H}, \mathrm{t}, J=7.1 \mathrm{~Hz}), 5.04$ $(1 \mathrm{H}, \mathrm{t}, J=7.1 \mathrm{~Hz}), 5.17(1 \mathrm{H}, \mathrm{t}, J=7.1 \mathrm{~Hz}) ;{ }^{13} \mathrm{C}-\mathrm{NMR}\left(\mathrm{CDCl}_{3}\right) \delta 25.6,27.3,44.9,52.3,74.7,119.2$ (q, $\left.J_{\mathrm{C}-\mathrm{F}}=329.8 \mathrm{~Hz}\right), 210.4 ;{ }^{19} \mathrm{~F}-\mathrm{NMR}\left(\mathrm{CDCl}_{3}\right) \delta-10.0(6 \mathrm{~F}, \mathrm{~s})$; MS (ESI-TOF) $m / z 477[\mathrm{M}+\mathrm{H}]^{+}$; HRMS calcd. for $\mathrm{C}_{15} \mathrm{H}_{23} \mathrm{~F}_{6} \mathrm{O}_{6} \mathrm{~S}_{2}[\mathrm{M}+\mathrm{H}]^{+}, 477.0840$; found, 477.0842.

4-(2,2-Bis(trifluoromethylsulfonyl)ethyl)-2,6-dimethylheptane-3,5-dione (4i). According to the general procedure, this compound was obtained in $80 \%$ yield $(62.4 \mathrm{mg}, 0.139 \mathrm{mmol})$ by the reaction of $\mathrm{Tf}_{2} \mathrm{CHCH}_{2} \mathrm{CHTf}_{2}(\mathbf{5}, 100 \mathrm{mg}, 0.175 \mathrm{mmol})$ with 2,6-dimethylheptane-3,5-dione (33 $\left.\mu \mathrm{L}, 0.19 \mathrm{mmol}\right)$ in $\mathrm{CH}_{2} \mathrm{Cl}_{2}(0.15 \mathrm{~mL})$ for $2.5 \mathrm{~h}$ at room temperature and the following bulb-to-bulb distillation $\left(150-165^{\circ} \mathrm{C}\right.$ at $5 \mathrm{mmHg}$ ). Colorless oil; IR (neat) $v 2979,2941,2880,1725,1469,1396,1213,1114$, 
1024, $689 \mathrm{~cm}^{-1} ;{ }^{1} \mathrm{H}-\mathrm{NMR}\left(\mathrm{CDCl}_{3}\right) \delta 1.14(6 \mathrm{H}, \mathrm{d}, J=6.7 \mathrm{~Hz}), 1.16(6 \mathrm{H}, \mathrm{d}, J=7.0 \mathrm{~Hz}), 2.69-2.82(4 \mathrm{H}$, $\mathrm{m}), 4.73(1 \mathrm{H}, \mathrm{t}, J=7.3 \mathrm{~Hz}), 5.32(1 \mathrm{H}, \mathrm{t}, J=6.9 \mathrm{~Hz}) ;{ }^{13} \mathrm{C}-\mathrm{NMR}\left(\mathrm{CDCl}_{3}\right) \delta 17.8,18.4,24.5,41.5,57.3$, 74.7, $119.2\left(\mathrm{q}, J_{\mathrm{C}-\mathrm{F}}=329.7 \mathrm{~Hz}\right), 208.1 ;{ }^{19} \mathrm{~F}-\mathrm{NMR}\left(\mathrm{CDCl}_{3}\right) \delta-10.3(6 \mathrm{~F}, \mathrm{~s})$; MS (ESI-TOF) $m / z 449$ $[\mathrm{M}+\mathrm{H}]^{+}$; HRMS calcd. for $\mathrm{C}_{13} \mathrm{H}_{19} \mathrm{~F}_{6} \mathrm{O}_{6} \mathrm{~S}_{2}[\mathrm{M}+\mathrm{H}]^{+}$, 449.0527; found, 449.0508.

Triethyl 3,3-bis(trifluoromethylsulfonyl)propane-1,1,1-tricarboxylate (4j). According to the general procedure, this compound was obtained in $88 \%$ yield $(183 \mathrm{mg}, 0.349 \mathrm{mmol})$ by the reaction of triethyl methanetricarboxylate $(92.2 \mathrm{mg}, 0.397 \mathrm{mmol})$ with $\mathrm{Tf}_{2} \mathrm{CHCH}_{2} \mathrm{CHTf}_{2}(\mathbf{5}, 295 \mathrm{mg}, 0.515 \mathrm{mmol})$ in acetonitrile $(0.40 \mathrm{~mL})$ at $80{ }^{\circ} \mathrm{C}$ for $13 \mathrm{~h}$ and the following removal of $\mathrm{Tf}_{2} \mathrm{CH}_{2}$ using Kugelrohr oven $\left(100{ }^{\circ} \mathrm{C}\right.$ at $5 \mathrm{mmHg}$ ). Colorless oil; IR (neat) $v$ 2988, 2943, 2911, 1745, 1393, 1220, 1110, 1018, 860, $701 \mathrm{~cm}^{-1} ;{ }^{1} \mathrm{H}-\mathrm{NMR}\left(\mathrm{CDCl}_{3}\right) \delta 1.28(9 \mathrm{H}, \mathrm{t}, J=7.2 \mathrm{~Hz}), 3.45(2 \mathrm{H}, \mathrm{d}, J=5.0 \mathrm{~Hz}), 4.27(6 \mathrm{H}, \mathrm{q}$, $J=7.2 \mathrm{~Hz}), 6.53(1 \mathrm{H}, \mathrm{t}, J=5.0 \mathrm{~Hz}) ;{ }^{13} \mathrm{C}-\mathrm{NMR}\left(\mathrm{CDCl}_{3}\right) \delta 13.6,27.3,62.1,63.5,73.9,119.3(\mathrm{q}$, $\left.J_{\mathrm{C}-\mathrm{F}}=330.2 \mathrm{~Hz}\right), 165.4 ;{ }^{19} \mathrm{~F}-\mathrm{NMR}\left(\mathrm{CDCl}_{3}\right) \delta-8.5(6 \mathrm{~F}, \mathrm{~s})$; MS (ESI-TOF) $m / z 525[\mathrm{M}+\mathrm{H}]^{+}$; HRMS calcd. for $\mathrm{C}_{14} \mathrm{H}_{19} \mathrm{~F}_{6} \mathrm{O}_{10} \mathrm{~S}_{2}[\mathrm{M}+\mathrm{H}]^{+}$, 525.0324; found, 525.0299.

\section{Conclusions}

In summary, we successfully found that $\delta$-oxo-1,1-bis(triflyl)alkanes are obtained in good to excellent yields by the reaction of enolizable carbonyls with $\mathrm{Tf}_{2} \mathrm{CHCH}_{2} \mathrm{CHTf}_{2}$ (5). NMR study of a solution of tetrasulfone 5 in $\mathrm{CDCl}_{3}$ revealed smooth formation of reactive 1,1-bis(triflyl)ethylene (3) in a reversible manner. On the basis of this reaction, incorporation of $\mathrm{Tf}_{2} \mathrm{CH}$ functionality into a wide range of 1,3-dicarbonyl compounds was realized. Furthermore, gas-phase acidities of some $\delta$-oxo-1,1-bis(triflyl)alkanes thus obtained were determined by the FT-ICR technique. The present work is a notable extension of our synthetic methodology for $\mathrm{Tf}_{2} \mathrm{CH}$ type carbon acids. Further studies on this reaction and catalysis of the $\delta$-oxo-1,1-bis(triflyl)alkanes are under progress in our laboratory.

\section{Acknowledgments}

This work was partially supported by a Grant-in-Aid for Scientific Research on Innovative Areas "Advanced Molecular Transformations by Organocatalysts" from the MEXT and by the Asahi Glass Foundation. $\mathrm{Tf}_{2} \mathrm{CH}_{2} 1$ was kindly provided from Central Glass Co., Ltd, Tokyo, Japan.

\section{Conflicts of Interest}

The authors declare no conflict of interest.

\section{References}

1. Leito, I.; Raamat, E.; Kütt, A.; Saame, J.; Kipper, K.; Koppel, I.A.; Koppel, I.; Zhang, M.; Mishima, M.; Yagupolskii, L.M.; et al. Revision of the gas-phase acidity scale below $300 \mathrm{kcal} \mathrm{mol}^{-1}$. J. Phys. Chem. A 2009, 113, 8421-8424.

2. Koppel, I.A.; Taft, R.W.; Anvia, F.; Zhu, S.-Z.; Hu, L.-Q.; Sung, K.-S.; DesMarteau, D.D.; Yagupolskii, L.M.; Yagupolskii, Y.L.; Ignat'ev, N.V.; et al. The gas-phase acidities of very strong neutral Brønsted acids. J. Am. Chem. Soc. 1994, 116, 3047-3057. 
3. Bordwell, F.G. Equilibrium acidities in dimethyl sulfoxide solution. Acc. Chem. Res. 1988, 21, 456-463.

4. Ishihara, K.; Hasegawa, A.; Yamamoto, H. Polystyrene-bound tetrafluorophenylbis(triflyl)methane as an organic-solvent-swellable and strong Brønsted acid catalyst. Angew. Chem. Int. Ed. 2001, 40, 4077-4079.

5. Hasegawa, A.; Ishikawa, T.; Ishihara, K.; Yamamoto, H. Facile Synthesis of aryl- and alkyl-bis(trifluoromethylsulfonyl)methanes. Bull. Chem. Soc. Jpn. 2005, 78, 1401-1410.

6. Hasegawa, A.; Naganawa, Y.; Fushimi, M.; Ishihara, K.; Yamamoto, H. Design of Brønsted acid-assisted chiral Brønsted acid catalyst bearing a bis(triflyl)methyl group for a Mannich-type reaction. Org. Lett. 2006, 8, 3175-3178.

7. Yanai, H.; Takahashi, A.; Taguchi, T. 1,4-Addition of silicon dienoates to $\alpha, \beta$-unsaturated aldehydes catalyzed by in situ-generated silicon Lewis acid. Chem. Commun. 2010, 46, 8728-8730.

8. Yanai, H.; Yoshino, Y.; Takahashi, A.; Taguchi, T. Carbon acid induced Mukaiyama aldol type reaction of sterically hindered ketones. J. Org. Chem. 2010, 75, 5375-5378.

9. Takahashi, A.; Yanai, H.; Zhang, M.; Sonoda, T.; Mishima, M.; Taguchi, T. Highly effective vinylogous Mukaiyama-Michael reaction catalyzed by silyl methide species generated from 1,1,3,3-tetrakis(trifluoromethanesulfonyl)propane. J. Org. Chem. 2010, 75, 1259-1265.

10. Takahashi, A.; Yanai, H.; Taguchi, T. Tetrakis(trifluoromethanesulfonyl)propane: Highly effective Brønsted acid catalyst for vinylogous Mukaiyama-Michael reaction of $\alpha, \beta$-enones with silyloxyfurans. Chem. Commun. 2008, 2385-2387.

11. Yanai, H.; Ogura, H.; Fukaya, H.; Kotani, A.; Kusu, F.; Taguchi, T. An effective method to introduce carbon acid functionality: 2,2-Bis(trifluoromethanesulfonyl)ethylation reaction of arenes. Chem. Eur. J. 2011, 17, 11747-11751.

12. Yanai, H.; Taguchi, T. Organic acid induced olefination reaction of lactone. Chem. Commun. 2012, 48, 8967-8969.

13. Yanai, H.; Ishii, N.; Matsumoto, T., Taguchi, T. Organic acid catalysis in reactions of lactones with silicon enolates, Asian J. Org. Chem. 2013, 2, 989-996.

14. Yanai, H.; Egawa, S.; Taguchi, T. Reductive alkylation of bis(triflyl)methane through self-promoting formation of easily isolable 1,1-bis(triflyl)alkenes. Tetrahedron Lett. 2013, 54, 2160-2163.

15. Koshar, R.J.; Barber, L.L. 1,1,3,3-Tetrakis(perfluoroalkylsulfonyl)propanes. U.S. Patent 4053519, 11 October 1977.

16. Yanai, H.; Fujita, M.; Taguchi, T. A regioselective synthesis of poly-substituted aryl triflones through self-promoting three component reaction. Chem. Commun. 2011, 47, 7245-7247.

17. Yanai, H.; Yoshino, T.; Fujita, M.; Fukaya, H.; Kotani, A.; Kusu, F.; Taguchi, T. Synthesis, characterization, and applications of zwitterions containing a carbanion moiety. Angew. Chem. Int. Ed. 2013, 52, 1560-1563.

18. Yanai, H.; Takahashi, Y.; Fukaya, H.; Dobashi, Y.; Matsumoto, T. 2-(Pyridinium-1-yl)-1,1bis(triflyl)ethanides: Structural behaviour and availability as bis(triflyl)ethylating reagents. Chem. Commun. 2013, 49, 10091-10093.

19. Lias, S.G.; Bartmess, J.E.; Liebman, J.F.; Holmes, J.L.; Levin, R.D.; Mallard, G.W. Gas-phase ion and neutral thermochemistry. J. Phys. Chem. Ref. Data 1988, 17 (Suppl. 1), 1-861. 
20. Zhang, M.; Badal, M.M.R.; Koppel, I.A. Mishima, M. Gas-phase acidities of $\alpha$ - and $\alpha$, $\alpha-\mathrm{SO}_{2} \mathrm{CF}_{3}$-substituted toluenes varying resonance demand in the electron-rich system. Bull. Chem. Soc. Jpn. 2013, 86, 813-820.

21. Takamura, K.; Fuse, T.; Arai, K.; Kusu, F. A review of a new voltammetric method for determining acids. J. Electroanal. Chem. 1999, 468, 53-63.

22. Kim, H.-S.; Chung, T.D.; Kim, H. Voltammetric determination of the $\mathrm{p} K_{\mathrm{a}}$ of various acids in polar aprotic solvents using 1,4-benzoquinone. J. Electroanal. Chem. 2001, 498, 209-215.

Sample Availability: Samples of the compounds are available from the authors.

(C) 2013 by the authors; licensee MDPI, Basel, Switzerland. This article is an open access article distributed under the terms and conditions of the Creative Commons Attribution license (http://creativecommons.org/licenses/by/3.0/). 